\title{
Atomic Force Microscopy Reveals Bistable Configurations of Dibenzo[a,h]thianthrene and their Interconversion Pathway
}

\author{
Niko Pavliček, ${ }^{1, *}$ Benoit Fleury, ${ }^{2, \dagger}$ Mathias Neu, ${ }^{1}$ Judith Niedenführ, ${ }^{1}$ Coral Herranz-Lancho, ${ }^{2}$ \\ Mario Ruben, ${ }^{2,3}$ and Jascha Repp ${ }^{1}$ \\ ${ }^{1}$ Institute of Experimental and Applied Physics, University of Regensburg, 93053 Regensburg, Germany \\ ${ }^{2}$ Institute of Nanotechnology (INT), Karlsruhe Institute of Technology (KIT), 76344 Eggenstein-Leopoldshafen, Germany \\ ${ }^{3}$ Institut de Physique et Chimie des Matériaux de Strasbourg (IMCMS), CNRS-Université de Strasbourg, 67034 Strasbourg, France
}

(Received 9 December 2011; published 23 February 2012)

We investigated dibenzo[a,h]thianthrene molecules adsorbed on ultrathin layers of $\mathrm{NaCl}$ using a combined low-temperature scanning tunneling and atomic force microscope. Two stable configurations exist corresponding to different isomers of free nonplanar molecules. By means of excitations from inelastic electron tunneling we can switch between both configurations. Atomic force microscopy with submolecular resolution allows unambiguous determination of the molecular geometry, and the pathway of the interconversion of the isomers. Our investigations also shed new light on contrast mechanisms in scanning tunneling microscopy.

DOI: 10.1103/PhysRevLett.108.086101

Recently, the chemical structure of a pentacene molecule has been visualized by means of noncontact atomic force microscopy (AFM) [1]. Shortly after, this method assisted in identifying the structure of an organic molecule [2]. In conjunction with the capability of scanning tunneling microscopy (STM) to perform orbital imaging on ultrathin insulating films [3], it is possible to gain independent and complementary information of the molecular as well as of the adsorption geometry, but also of the electronic structure of individual molecules.

Unambiguous identification of configurational changes of adsorbed molecules is a challenging task by means of STM alone [4], probing the local density of states rather than geometry. Usually, additional techniques such as nearedge $\mathrm{x}$-ray adsorption fine structure measurements have to be employed $[5,6]$.

In this Letter, we present combined STM and AFM experiments of dibenzo[a,h]thianthrene (DBTH) molecules adsorbed on ultrathin layers of sodium chloride. We demonstrate controlled switching between two different molecular configurations by means of inelastic excitations. AFM images with submolecular resolution directly reveal the configurational changes. Stereochemistry could be utilized to determine their interconversion pathway in detail.

All AFM measurements were carried out in a homebuilt combined STM and AFM operating in an ultrahigh vacuum $\left(p<10^{-10} \mathrm{mbar}\right)$ at $T=5 \mathrm{~K}$. The AFM, based on the qPlus tuning fork design (spring constant $k_{0} \approx$ $1.8 \times 10^{3} \mathrm{~N} \mathrm{~m}^{-1}$, resonance frequency $f_{0}=26057 \mathrm{~Hz}$, quality factor $Q \approx 10^{4}$ ) [7], was operated in the frequency modulation mode [8]. Sub-Ångstrom oscillation amplitudes have been used to maximize the lateral resolution [9]. Some of the STM measurements (Figs. 1 and 2) were performed in a similar modified commercial STM from
PACS numbers: 68.43.Fg, 68.37.Ef, 68.37.Ps, 82.37.Gk

SPS-CreaTec. The bias voltage $V$ was applied to the sample.

Sodium chloride was evaporated onto clean $\mathrm{Cu}(111)$ single crystals at sample temperatures of about $280 \mathrm{~K}$ [10]. All experiments were carried out on a double layer, and we denote this substrate system as $\mathrm{NaCl}(2 \mathrm{ML}) /$ $\mathrm{Cu}(111)$. The DBTH molecules were synthesized as described previously [11].

Low coverages of $\mathrm{CO}$ (for tip functionalization) and DBTH molecules were adsorbed at sample temperatures below $10 \mathrm{~K}$. Following a recently developed technique, the tip had been terminated with a CO molecule for all AFM measurements to enhance the resolution considerably [1]. (a)

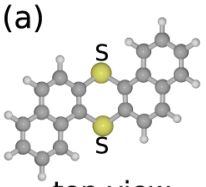

top view

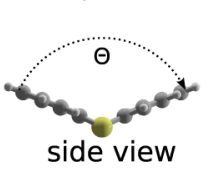

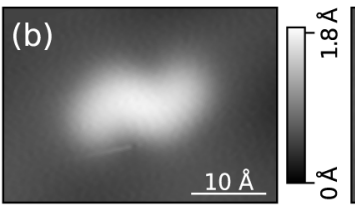

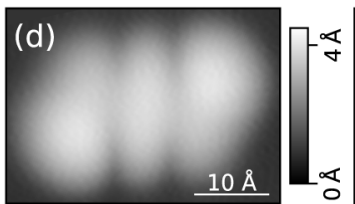

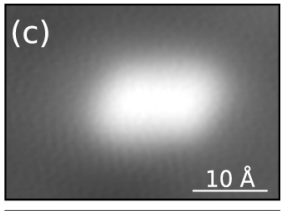

(e)
FIG. 1 (color online). Bias dependent STM imaging reveals two possible nonequivalent species of the same individual DBTH molecule on $\mathrm{NaCl}(2 \mathrm{ML}) / \mathrm{Cu}(111)$. (a) Ball and stick model of DBTH molecules and the definition of dihedral angle $\Theta$. Small white, medium grey, and big yellow balls represent $\mathrm{H}, \mathrm{C}$, and S atoms. (b) and (c) Constant-current images of $U$ and $D$ configurations, respectively, at low bias voltage (imaging parameters: $I=0.4 \mathrm{pA}, V=0.01 \mathrm{~V})$. (d) and (e) Images corresponding to the lowest unoccupied molecular orbital acquired with the same metal tip apex $(I=0.2 \mathrm{pA}, V=2.3 \mathrm{~V})$. 

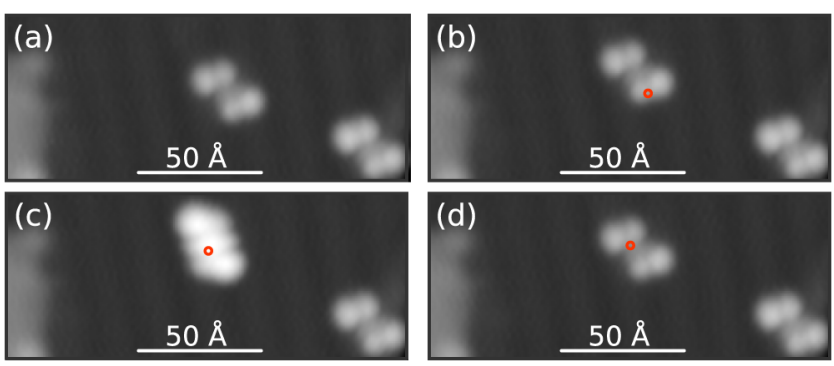

FIG. 2 (color online). Switching between molecular configurations by means of inelastic excitations. A manipulation sequence shows subsequent STM orbital images $(I=0.2 \mathrm{pA}$, $V=2.35 \mathrm{~V})$. Circles indicate previous positions of the center of the molecule. From (a) to (b) only the adsorption position changes. In contrast, both position and in-plane orientation change from (b) to (c) and subsequently to (d).

DBTH molecules are within the family of thianthrenes, in which, due to the presence of the lone pairs of the thioether groups, the molecules are folded along the S-S axis [12-17]. To obtain the folding angle for DBTH molecules, we have performed density functional theory calculations $[18,19]$ for a free molecule (i.e., without substrate) using the highly optimized CPMD code [20]. Ab initio norm-conserving pseudopotentials [21] were used. In the optimized geometry, we find a dihedral angle [see Fig. 1(a) for definition] of $\Theta=133^{\circ}$ [22]. This value is larger than the one observed for thianthrene by x-ray crystallography with $\Theta=128^{\circ}$ [13], whereby the difference can be attributed to the extension of the $\pi$ conjugation in DBTH molecules and thus flattening the molecule.

Bias dependent STM images of DBTH on $\mathrm{NaCl}(2 \mathrm{ML})$ / $\mathrm{Cu}(111)$ are presented in Fig. 1. Two species of DBTH molecules that are nonequivalent with respect to translation, mirroring, and/or rotations exist on the surface, which we denote as $U$ and $D$ as indicated in the caption of Fig. 1. STM images at low bias voltages [Figs. 1(b) and 1(c)] as well as orbital images [Figs. 1(d) and 1(e)] show distinct differences between both species. However, we note that the nodal plane structure of the orbital images is the same. In addition, STS spectra acquired on both species are similar, and the only peak in the accessible voltage range at around $2.45 \mathrm{~V}$ corresponds to the negative ion resonance.

By means of inelastic excitations $[23,24]$ it is possible to induce the lateral motion of individual molecules, as well as to switch between the two species. The results are presented in Fig. 2 [25]. An analysis of many excitations reveals that whenever a molecule switches from $U$ to $D$ or vice versa, both the in-surface-plane orientation and the adsorption site change (see below for a detailed adsorption geometry determination). Thus, every species can be assigned to a different adsorption site.

STM images at low bias voltages demonstrate that neither species has an influence on the scattering wave pattern of the substrate's interface state. Hence, both species are neutral $[26,27]$. The similarity in the electronic structure (nodal planes in orbital imaging and the energy of the negative ion resonance) suggests that the molecule is either in the same or an equivalent configuration, that is, it has a similar dihedral angle. The different appearance could be simply due to different adsorption sites leading to slightly different electronic properties. It must be emphasized that we cannot draw any conclusions from the STM data going beyond what has been discussed until this point.

As a next step, we performed constant height $\Delta f$ imaging presented in Fig. 3(a) in the AFM mode. It is immediately apparent that the two species correspond to different molecular configurations [28]. First, consider the S-S axis. Whereas $U$ molecules show a characteristic bright stripe, $D$ molecules do not show any atomic contrast at the center at the same tip height. In addition, the carbon rings of $U$ molecules show an apparent distortion.

Before we shed light on the actual structure of both configurations, we have to discuss the origin of the contrast in the frequency shift. For the small amplitudes used here, the frequency shift well approximates the force gradient. While attractive long-range forces are responsible for overall negative $\Delta f$ background, repulsive short-range contributions due to Pauli repulsion are decisive for the intramolecular contrast as was shown in $[1,29]$. These short-range forces are very sensitive to the tip-molecule distance. An examination of $\mathrm{C}$ atoms and $\mathrm{C}-\mathrm{C}$ bonds shows a specific gradient for both species. In the $D(U)$ configuration the contrast increases (decreases) with the distance to the S-S axis. As discussed above, in this regime we can attribute a higher contrast with stronger repulsive contributions, and consequently a smaller distance to the molecule. Thus, the bright band along the S-S axis in the $U$ configuration can be attributed to a small distance to the tip. We believe that the sharp feature is a fingerprint of the CO molecule at the tip apex which bends due to the protruding S atoms [29]. Accordingly, in this configuration the $\mathrm{S}$ atoms are pointing upward, and the aromatic rings are close to the surface. In contrast, the $\mathrm{S}$ atoms of $D$ molecules are closest to the surface, whereas its naphthalene units are pointing upwards. Both configurations are illustrated in Fig. 3(d).

The stereochemistry of DBTH molecules can be utilized to determine the pathway of the configurational change $[30,31]$. We have estimated the barrier for flapping to be about $200 \mathrm{meV}$ from our density functional theory calculations using the relative energy vs folding angle $\Theta$. For this purpose, we optimized the geometry for fixed $\Theta$ in steps of $10^{\circ}$. As the flapping of molecules is frozen at the temperatures of our experiments, there are two enantiomers of the free molecule possessing $C_{2}$ symmetry, as depicted in Fig. 3(e) [32]. In general, going from upward to downward pointing $\mathrm{S}$ atoms can be realized either by flipping from one face to the other, or by flapping the naphthalene units with respect to the $\mathrm{S}$ atoms. The 

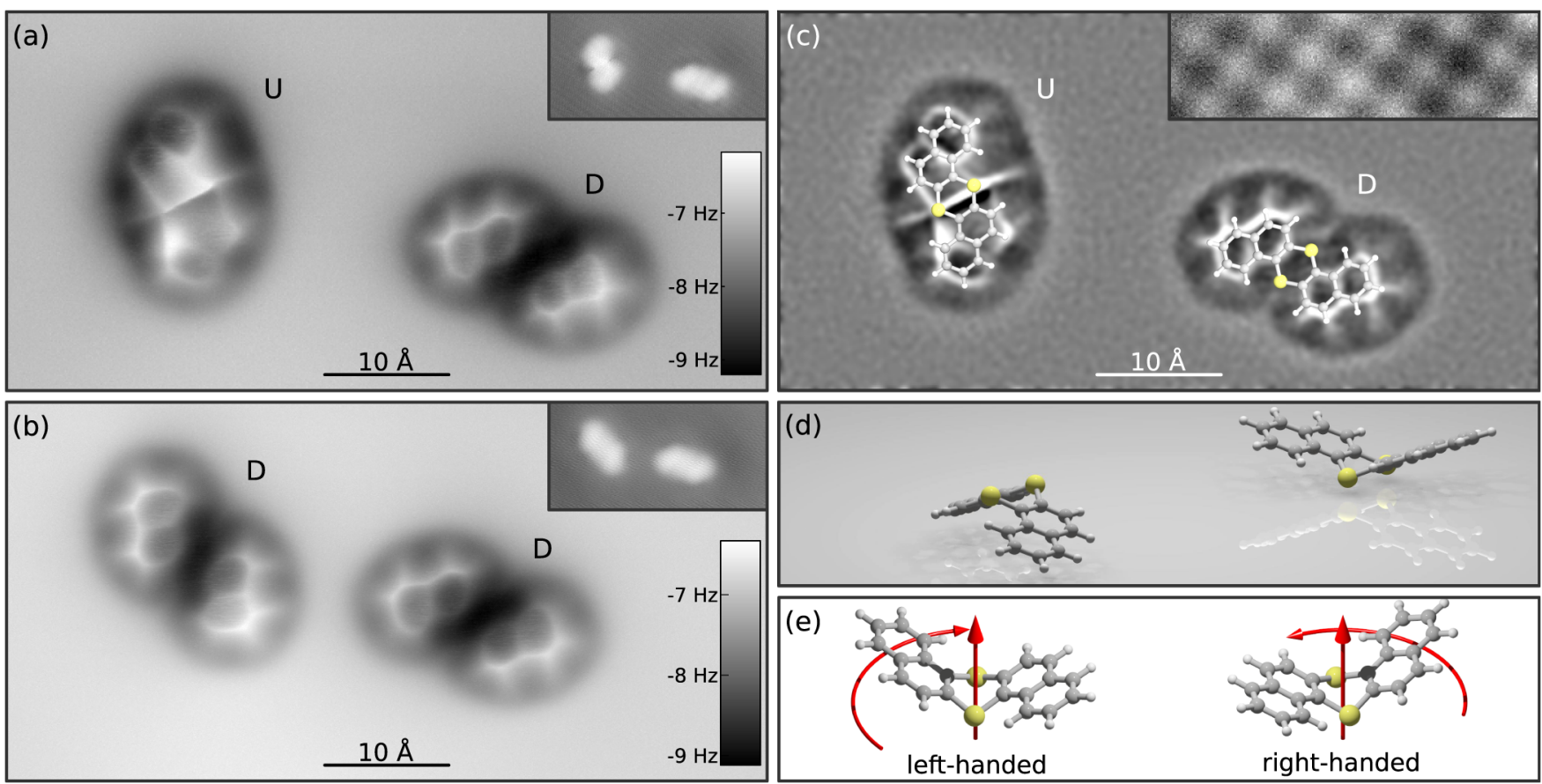

FIG. 3 (color online). AFM measurements on $\mathrm{DBTH}$ on $\mathrm{NaCl}(2 \mathrm{ML}) / \mathrm{Cu}(111)$ with a CO-functionalized tip. (a) Constant height AFM image. Imaging parameters: oscillation amplitude $A=0.5 \AA, V=0 \mathrm{~V}, \Delta z=0.0 \AA$. $\Delta z$ corresponds to a distance decrease with respect to an STM set point of $I=0.5 \mathrm{pA}, V=0.4 \mathrm{~V}$ above the clean $\mathrm{NaCl}(2 \mathrm{ML}) / \mathrm{Cu}(111)$. (b) Image of the same area as in (a) after both molecules changed their adsorption position $(A=0.5 \AA, V=0 \mathrm{~V}, \Delta z=0.1 \AA$ ). Insets in (a) and (b) show constant-current STM images of the same frame. Panel (c) represents the curvature of the image in (a) obtained by calculating the Laplacian. Molecular models (drawn to scale) for $U$ and $D$ are overlaid as a guide to the eye; the slightly larger appearance of molecules has been discussed previously [29]. The inset shows an atomically resolved $\mathrm{NaCl}$ lattice. (d) Model representing molecules in $U$ and $D$ configuration on a surface. (e) Model depicting chiral enantiomers of the free molecule.
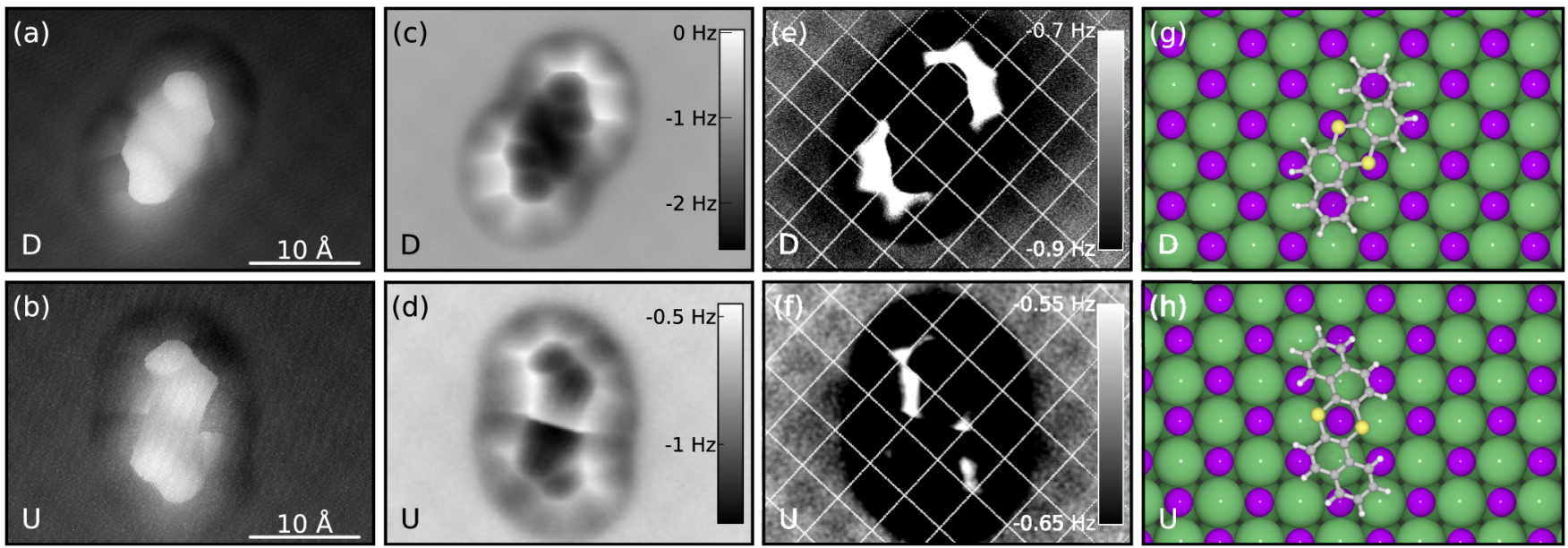

FIG. 4 (color online). Determination of the molecular adsorption position for down (top row) and up (bottom) geometry using AFM with STM feedback. (a) and (b) Constant-current STM images (imaging parameters $I=1.0 \mathrm{pA}, V=0.3 \mathrm{~V}$ ). The corresponding frequency shift $\Delta f$ recorded simultaneously is shown in panels (c) and (d) (oscillation amplitude $A=0.5 \AA$, and $A=1.0 \AA$, respectively). Panels (e) and (f) show the same data with a different $\Delta f$ scale to highlight the faint maxima resembling $\mathrm{Cl}$ sites, for which a lattice was overlaid as a guide to the eye. Panels ( $g$ ) and (h) show models of the adsorption position as determined from the experimental data. Big green and small purple balls represent $\mathrm{Cl}^{-}$and $\mathrm{Na}^{+}$ions, respectively. 
molecular structure projected onto the surface plane would change for flipping, but not for flapping. As can be seen from the left molecule in Figs. 3(a) and 3(b), the latter is the case. From this it follows immediately that switching is caused by a change of the folding angle, and the initial and final configurations correspond to the different chiral enantiomers of the free molecule as depicted in Fig. 3(e).

To get more insight, we determined the adsorption site of molecules in both states. Therefore, we used AFM imaging with STM constant-current feedback. That is, the frequency shift $\Delta f$ is recorded simultaneously with the STM topography while the STM feedback is used to control the tip height [33]. Figures 4(c)-4(f) show the frequency shift $\Delta f$ of such measurements for both configurations. Recently, it has been shown that the faint maxima correspond to the $\mathrm{Cl}$ sites of the $\mathrm{NaCl}(100)$ lattice [2]. From the models of the adsorption geometries shown in Figs. 4(g) and 4(h) we can conclude that the S atoms are located on top of Na sites for $D$ molecules. This can be rationalized by the ionic nature of the $\mathrm{NaCl}$ lattice as follows: The downward pointing lone pairs of $\mathrm{S}$ atoms are attracted by $\mathrm{Na}^{+}$ions and repelled by $\mathrm{Cl}^{-}$ions. In contrast, the $\mathrm{S}$ atoms of $U$ molecules are farthest from the surface, and the naphthalene units align with a polar direction of $\mathrm{NaCl}$. This results in a geometry in which the center of the molecule is located above a $\mathrm{Na}$ site [34].

Since we are by now in a position, in which we have a detailed picture of our system, we would like to revisit Fig. 1 particularly with regard to the actual molecular structure. Resonance images resembling the orbital structure cannot be used to make a statement about the geometry. In contrast, images at low voltages can be argued to be due to modifications of the tunneling barrier and should therefore be somewhat related to geometry. However, these images show a pronounced $s$ shape for $U$ and a relatively straight rod for $D$ molecules, whereas the apparent height above their center is the same. Thus, they neither reflect the lateral geometry nor the vertical changes in the structure.

In conclusion, we have revealed the exact geometry of two different configurations of DBTH molecules on $\mathrm{NaCl}(2 \mathrm{ML}) / \mathrm{Cu}(111)$ using combined STM and AFM experiments. Our experiments demonstrate reproducible and nondestructive switching between both configurations. We have shown that AFM with submolecular resolution is capable of directly revealing configurational changes in real space. Our data visualize displacements perpendicular to the surface in a nonplanar molecule. Taking the chirality of DBTH molecules into account, we could unambiguously determine the interconversion pathway.

The authors thank L. Gross, N. Moll, G. Meyer, I. Swart, and F. Albrecht for valuable comments, and F. J. Giessibl for instrumentation support. Financial support from the
Volkswagen Foundation (Lichtenberg program), the Deutsche Forschungsgemeinschaft (GRK 1570 and SPP 1243), and from the Marie-Curie-ITN SMALL is gratefully acknowledged.

*niko.pavlicek@physik.uni-regensburg.de

${ }^{\dagger}$ On leave from Institut Parisien de Chimie Moléculaire, UPMC and CNRS UMR7201, UPMC-Paris 6, 75252, Paris Cedex 05, France.

[1] L. Gross, F. Mohn, N. Moll, P. Liljeroth, and G. Meyer, Science 325, 1110 (2009).

[2] L. Gross, F. Mohn, N. Moll, G. Meyer, R. Ebel, W. M. Abdel-Mageed, and M. Jaspars, Nature Chem. 2, 821 (2010).

[3] J. Repp, G. Meyer, S. M. Stojković, A. Gourdon, and C. Joachim, Phys. Rev. Lett. 94, 026803 (2005).

[4] T. A. Jung, R. R. Schlittler, and J. K. Gimzewski, Nature (London) 386, 696 (1997).

[5] W. Auwärter, F. Klappenberger, A. Weber-Bargioni, A. Schiffrin, T. Strunskus, C. Wöll, Y. Pennec, A. Riemann, and J. V. Barth, J. Am. Chem. Soc. 129, 11279 (2007).

[6] A. Weber-Bargioni, W. Auwärter, F. Klappenberger, J. Reichert, S. Lefrançois, T. Strunskus, C. Wöll, A. Schiffrin, Y. Pennec, and J. V. Barth, Chem. Phys. Chem. 9, 89 (2008).

[7] F. J. Giessibl, Appl. Phys. Lett. 76, 1470 (2000).

[8] T. R. Albrecht, P. Grütter, D. Horne, and D. Rugar, J. Appl. Phys. 69, 668 (1991).

[9] F. J. Giessibl, Rev. Mod. Phys. 75, 949 (2003).

[10] R. Bennewitz, V. Barwich, M. Bammerlin, C. Loppacher, M. Guggisberg, A. Baratoff, E. Meyer, and H.-J. Güntherodt, Surf. Sci. 438, 289 (1999).

[11] A. Spurg, G. Schnakenburg, and S. R. Waldvogel, Chem. Eur. J. 15, 13313 (2009).

[12] I. Rowe and B. Post, Acta Crystallogr. 11, 372 (1958).

[13] H. Lynton and E. G. Cox, J. Chem. Soc. 4886 (1956).

[14] J. Stenhouse, Justus Liebigs Annalen der Chemie 149, 247 (1869).

[15] M. J. Aroney, R. J. W. Le Fevre, and J. D. Saxby, J. Chem. Soc. 571 (1965).

[16] K. L. Gallaher and S.H. Bauer, J. Chem. Soc., Faraday Trans. 2 71, 1173 (1975).

[17] S. Hosoya, Acta Crystallogr. 16, 310 (1963).

[18] P. Hohenberg and W. Kohn, Phys. Rev. 136, B864 (1964).

[19] W. Kohn and L. J. Sham, Phys. Rev. 140, A1133 (1965).

[20] CPMD V3.15 Copyright IBM Corp 1990-2011, Copyright MPI fuer Festkoerperforschung Stuttgart 1997-2001, http://www.cpmd.org.

[21] J. P. Perdew, K. Burke, and M. Ernzerhof, Phys. Rev. Lett. 77, 3865 (1996).

[22] The cell size was $18 \AA \times 16 \AA \times 9 \AA$. A plane-wave cutoff energy of 130 Ry was used.

[23] T. Komeda, Y. Kim, M. Kawai, B. N. J. Persson, and H. Ueba, Science 295, 2055 (2002).

[24] T. Sonnleitner, I. Swart, N. Pavliček, A. Pöllmann, and J. Repp, Phys. Rev. Lett. 107, 186103 (2011). 
[25] STM and AFM data before and after an excitation show that molecules and $\mathrm{NaCl}$ remain undamaged.

[26] J. Repp, G. Meyer, F. E. Olsson, and M. Persson, Science 305, 493 (2004).

[27] I. Swart, T. Sonnleitner, and J. Repp, Nano Lett. 11, 1580 (2011).

[28] C. Loppacher, M. Guggisberg, O. Pfeiffer, E. Meyer, M. Bammerlin, R. Lüthi, R. Schlittler, J. K. Gimzewski, H. Tang, and C. Joachim, Phys. Rev. Lett. 90, 066107 (2003).

[29] N. Moll, L. Gross, F. Mohn, A. Curioni, and G. Meyer, New J. Phys. 12, 125020 (2010).

[30] R. Raval, Chem. Soc. Rev. 38, 707 (2009).

[31] M. J. Comstock, D. A. Strubbe, L. Berbil-Bautista, N. Levy, J. Cho, D. Poulsen, J. M. J. Fréchet, S. G.
Louie, and M. F. Crommie, Phys. Rev. Lett. 104, 178301 (2010).

[32] In total, 4 configurational isomers exist on the surface: one pair of enantiomers for molecules in each configuration $(U$ and $D)$.

[33] STM and AFM signals are convoluted in this mode. However, this mode enables atomic resolution on $\mathrm{NaCl}$ and stable imaging of the molecules at the same time [2].

[34] All possible, independently determined adsorption geometries are consistent with symmetry considerations. We estimate the error bars to be $\pm 0.5 \AA$ for the site determination and $\pm 3^{\circ}$ for the in-plane orientation. 